clinical variables independently associated with BMD were determined by linear regression.

Results: A total of 230 patients were recruited including 65 IIMs, 65 non-rheumatological controls, 50 RA and 50 SLE patients. The mean age was $58.6 \pm 10.96$ years and $76.9 \%$ were female. There was no significant difference on demographics between the two groups. Almost all IIMs patients (98\%) and 52\% of controls had exposed to GCs ( $<<0.001)$. Significantly more IIMs patients had used immunosuppressants (92.3\% vs $38.5 \%, p<0.001)$ and biologics $(13.8 \%$ vs $1.5 \%, p=0.01)$. The prevalence of reduced $\mathrm{BMD}$ and osteoporosis were significantly higher in IIMs patients than non-rheumatological control (Reduced BMD: 73.8\% vs 43.1\%, p=0.043; Osteoporosis: $29.2 \%$ vs $13.8 \%, p=0.033$ ) (Table 1 ). The mean lumbar spine and hip BMD were $0.886 \pm 0.181 \mathrm{~g} / \mathrm{cm}^{2}$ and $0.651 \pm 0.144 \mathrm{~g} / \mathrm{cm}^{2}$ in IIMs patients, which were significantly lower than that of the control group $\left(0.960 \pm 0.143 \mathrm{~g} / \mathrm{cm}^{2}, \mathrm{p}=0.011\right.$ and $0.751 \pm 0.127 \mathrm{~g} /$ $\mathrm{cm}^{2}, \mathrm{p}<0.001$ ) (Figure 1). Multivariate analysis confirmed IIMs were associated with increased risk of reduced BMD (Odds ratio:2.118, $\mathrm{p}=0.048,95 \% \mathrm{Cl} 1.005-4.461$ ). The prevalence of reduced $\mathrm{BMD}$ and osteoporosis were not significantly different between IIMs, RA and SLE patients but the mean hip BMD was the lowest in the IIM group $\left(0.641 \pm 0.152 \mathrm{~g} / \mathrm{cm}^{2}\right.$ vs $0.663 \pm 0.102 \mathrm{~g} / \mathrm{cm}^{2}$ in the RA group vs $0.708 \pm 0.132 \mathrm{~g}$ / $\mathrm{cm}^{2}$ in the SLE group, $\left.\mathrm{p}=0.035\right)$. Lower BMI $(\mathrm{p}=0.035)$ and more advanced age $(p<0.001)$ were associated with lower BMD in the IIM patients.

Conclusion: Reduced BMD was more prevalent in IIM patients than non-rheumatological controls. Lower BMI and more advanced age were associated with lower BMD. Vigilant monitoring of BMD and use of antiresorptive treatment should be considered in IIM patients.

REFERENCES:

[1] Briot K, Geusens P, Em Bultink I et al. Inflammatory diseases and bone fragility. Osteoporos Int. 2017;28:3301-14.

[2] So H, Yip ML, Wong AKM. Prevalence and associated factors of reduced bone mineral density in patients with idiopathic inflammatory myopathies. Int J Rheum Dis. 2016;19:521-8.

Table 1. Prevalence of reduced BMD in IIM patients and non-rheumatologcial controls

\begin{tabular}{llll}
\hline & Myositis $(\mathrm{n}=65)$ & $\begin{array}{l}\text { Non-rheumatological } \\
\text { controls }(\mathrm{n}=65)\end{array}$ & Significance \\
\hline Osteopenia at LS & $25(38.5 \%)$ & $20(30.8 \%)$ & 0.357 \\
Osteoporosis at LS & $13(20 \%)$ & $6(9.2 \%)$ & 0.082 \\
Osteopenia at NOF & $29(44.6 \%)$ & $26(40 \%)$ & 0.542 \\
Osteoporosis at NOF & $12(18.5 \%)$ & $6(9.2 \%)$ & 0.119 \\
Osteopenia overall & $29(44.6 \%)$ & $28(43.1 \%)$ & 0.860 \\
Osteoporosis overall & $19(29.2 \%)$ & $9(13.8 \% \%)$ & 0.033 \\
Reduced BMD overall & $48(73.8 \%)$ & $37(56.9 \%)$ & 0.043 \\
Occurrence of fragility fracture None $=52$ & None=54 & 0.651 \\
& Vertebral $=5$ & Vertebral=4 & \\
& Femoral=1Femoral=1 & Femoral=0 & \\
& Wrist=4 & Wrist $=3$ & \\
& Other sites $=3$ & Other sites=3 & \\
\hline
\end{tabular}

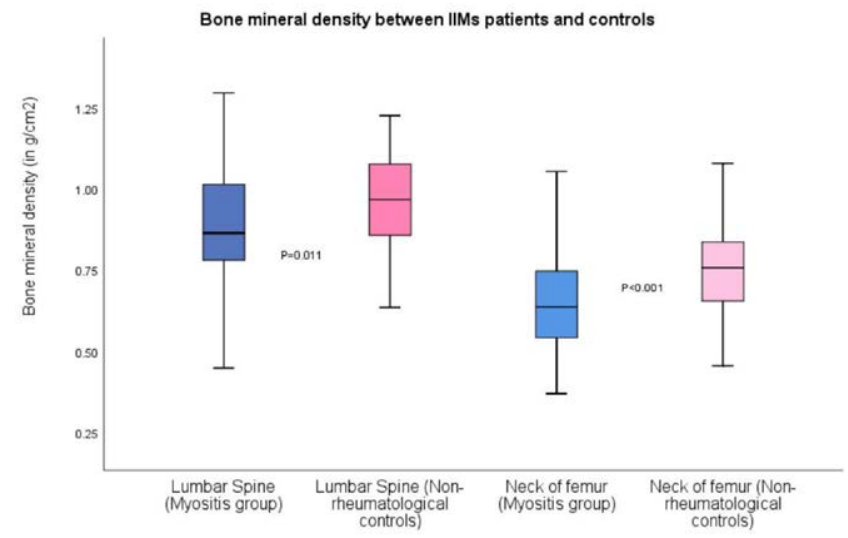

Disclosure of Interests: None declared

DOI: 10.1136/annrheumdis-2021-eular.2824

\section{POS0866 \\ TWO-DIMENSIONAL HRCT-BASED RADIOMIC FEATURES IN SSC-ILD DISTINGUISH DRUG RESPONDERS FROM NON-RESPONDERS}

C. Meier ${ }^{1}$, M. Maciukiewicz ${ }^{1}$, M. Brunner ${ }^{1}$, J. Schniering ${ }^{1}$, H. Gabrys ${ }^{2}$, A. Kühnis ${ }^{2}$, O. Distler ${ }^{1}$, T. Frauenfelder ${ }^{3}$, S. Tanadini-Lang ${ }^{2}$, B. Maurer ${ }^{1}$. ${ }^{1}$ University of Zurich, University Hospital Zurich, Department of Rheumatology, Center of Experimental Rheumatology, Zurich, Switzerland; ${ }^{2}$ University of
Zurich, University Hospital Zurich, Department of Radiation Oncology, Zurich, Switzerland; ${ }^{3}$ University of Zurich, University Hospital Zurich, Institute for Diagnostic and Interventional Radiology, Zurich, Switzerland

Background: Management of patients with systemic sclerosis-associated interstitial lung disease (SSc-ILD) is complicated by high inter-patient variability. To date, no validated predictors of treatment response are available for routine use. High resolution computed tomography (HRCT)-based radiomics, i.e. the high-dimensional, quantitative analysis of imaging metadata, have previously been shown to be successful in discriminating (SSc-)ILD phenotypes in preclinical and clinical studies ${ }^{1}$. Since HRCT is an integral part of the routine work-up in SSc, HRCT-based radiomic features may hold potential as non-invasive biomarkers.

Objectives: To predict treatment response using two-dimensional (2D) HRCT-based radiomics in SSc-ILD patients from a prospectively followed cohort.

Methods: Inclusion criteria were diagnosis of SSc-ILD in HRCT, availability of a suitable chest HRCT scan within 12 months prior to initiation of a new treatment, and availability of clinical baseline and follow-up information. Treatment response was defined as the absence of all of the following over a follow-up period of 12-24 months: relative decrease in forced vital capacity (FVC) $\geq 5 \%$, increase of ILD in $\mathrm{HRCT}$ as assessed by a radiologist, change in treatment regimen due to insufficient response, ILD-related death or lung transplantation. Of each pre-treatment HRCT, 6 slices $(15 \pm 5 \mathrm{~mm}$ apart, starting from the basal lung margin) were manually segmented and $15132 \mathrm{D}$ radiomic features were extracted using the in-house software Z-Rad (Python 2.7). Features were Z-score transformed and pre-filtered for inter- and intra-reader robustness (intraclass correlation coefficient $>0.85$ ) and inter-feature correlation (Spearman's rho <0.9). A categorical linear regression model was created using 3 -fold cross-validated elastic nets for feature selection. Features were then summarized and divided by their number. For generation of a score cut-off, Youden's score was used. For two-group analyses of continuous variables, Wilcoxon's test was performed, whereas categorical data was assessed using Fisher's exact test.

Results: A total of 64 pre-treatment HRCTs from 54 patients were analyzed. In 9 patients, $>1$ asynchronous treatments were assessed, while 45 patients had only 1 eligible treatment approach. The response rate within the assessed follow-up period was $45.3 \%(n=29)$. For score generation, 13 radiomic features were selected and an optimal cut-off value of -0.1589 was determined. Univariate linear regression showed significant association between our categorical radiomics-based score and treatment response $(\mathrm{p}=0.007$, area under the curve $=0.65(0.51-0.79)$, sensitivity $=0.90$, specificity $=0.43)$, whereby a high score was predictive for treatment response.

No differences between patients with high $(n=46)$ or low $(n=18)$ scores were detected for baseline age (mean $\pm S D=55.5 \pm 12.0$ and $55.5 \pm 13.6$ years, $p=0.84$ ), duration of $S S c$ (mean $\pm S D=6.2 \pm 8.4$ and $4.7 \pm 4.4$ years, $p=0.79$ ), time since ILD diagnosis $(2.7 \pm 2.9$ and $2.4 \pm 3.1$ years, $\mathrm{p}=0.59)$, $\mathrm{FVC}(77.6 \pm 20.6$ and $80.1 \pm 17.9$ $\mathrm{p}=0.41)$ or DLco $(54.4 \pm 21.0$ and $57.6 \pm 18.9, \mathrm{p}=0.40)$. Distribution of anti-Scl-70 positivity ( $45.7 \%$ vs. $55.6 \%, p=0.58)$ and diffuse cutaneous disease $(47.7 \%$ vs $61.1 \%, p=0.41$ ) was not significantly different between patients with high and low scores, respectively, although a trend towards higher percentages in the high score group was observed.

Conclusion: Our results indicate that, following validation in external cohorts, radiomics may be a promising tool for future pre-treatment patient stratification. Moreover, our radiomics-based score seems not to be associated with commonly studied clinical predictors such as anti-Scl-70 positivity or lung function, underlining a possible additive value to 'traditional' clinical parameters.

REFERENCES:

[1] Schniering, J., et al. Resolving phenotypic and prognostic differences in interstitial lung disease related to systemic sclerosis by computed tomography-based radiomics. medRxiv [Preprint] doi:10.1101/2020.06.09.20124800 (2020).

Disclosure of Interests: Chantal Meier: None declared, Malgorzata Maciukiewicz: None declared, Matthias Brunner: None declared, Janine Schniering: None declared, Hubert Gabrys: None declared, Anja Kühnis: None declared, Oliver Distler Speakers bureau: Speaker fee on Scleroderma and related complications: Bayer, Boehringer Ingelheim, Medscape, Novartis, Roche. Speaker fee on rheumatology topic other than Scleroderma: MSD, iQone, Novartis, Pfizer, Roche, Consultant of: Consultancy fee for Scleroderma and its complications: Abbvie, Acceleron Pharma, Amgen, AnaMar, Arxx Therapeutics, Bayer, Baecon Discovery, Boehringer, CSL Behring, ChemomAb, Corbus Pharmaceuticals, Horizon Pharmaceuticals, Galapagos NV, GSK, Glenmark Pharmaceuticals, Inventiva, Italfarmaco, iQvia, Kymera, Medac, Medscape, Mitsubishi Tanabe Pharma, MSD, Roche, Roivant Sciences, Sanofi, UCB. Consultancy fee for rheumatology topic other than Scleroderma: Abbvie, Amgen, Lilly, Pfizer, Grant/research support from: Research Grants to investigate the pathophysiology and potential treatment 
of Scleroderma and its complications: Kymera Therapeutics, Mitsubishi Tanabe, Thomas Frauenfelder: None declared, Stephanie Tanadini-Lang: None declared, Britta Maurer Speakers bureau: Speaker fees from Boehringer-Ingelheim, Grant/ research support from: Grant/research support from AbbVie, Protagen, Novartis Biomedical Research, congress support from Pfizer, Roche, Actelion, mepha, and MSD

DOI: 10.1136/annrheumdis-2021-eular.2884

\section{POS0867 ALTERATION OF DUODENAL HISTOLOGY IN SYSTEMIC SCLEROSIS PATIENTS AFTER FECAL MICROBIOTA TRANSPLANTATION}

H. Didriksen ${ }^{1}$, N. Strahm ${ }^{2}$, Ø. Molberg ${ }^{1}$, H. Fretheim ${ }^{1}$, T. Garen ${ }^{1}$, Ø. Midtvedt ${ }^{1}$, O. Distler ${ }^{2}$, G. Kania ${ }^{2}$, A. M. Hoffmann-Vold ${ }^{1} .{ }^{1}$ Oslo universitetssykehus Rikshospitalet, Rheumatology, Oslo, Norway; ${ }^{2}$ University Hospital of Zürich, Rheumatology, Zürich, Switzerland

Background: Systemic sclerosis (SSc) is a complex autoimmune, multi-organ disease with gastro-intestinal tract (GIT) involvement significantly contributing to comorbidity. While understanding of mechanisms behind SSc-related GIT disease is incomplete, recent work shows that altered gut microbiota (dysbiosis) is present in many patients and associates with specific GIT symptoms. Intending to improve dysbiosis, we set up the controlled ReSScue pilot trial, where fecal microbiota transplantation (FMT) was conducted by duodenal infusions of Anaerobic Cultivated Human Intestinal Microbiome (ACHIM) or placebo. The results indicated that FMT by ACHIM induced short-term improvement on patient reported GIT symptoms, as evaluated by the validated UCLA GIT score (1). Duodenal biopsies were taken before and after FMT to assess potential effects of ACHIM on small intestinal mucosa.

Objectives: Assessment of duodenal histology and GIT symptoms before and after FMT by ACHIM compared to placebo.

Methods: In this explorative study, we assessed duodenal biopsies collected prior to first FMT (week 0), prior to second FMT (week 2) and at study end (week 16) from ReSScue trial patients who either received ACHIM $(n=5)$ or placebo $(n=4)$. To examine potential effects of FMT on the duodenal mucosa, we performed immunohistochemistry (IHC) staining on paraffin-embedded tissue samples using the following markers: Sirius red (SIR) for collagen fibers and monoclonal antibodies against gp38 (podoplanin, as marker for lymphatic vessels and fibroblasts), CD38 (as preliminary marker for adaptive immune cells) and CD64 (as preliminary marker for innate immune cells). We determined staining per area in the individual tissue slides for each marker using ImageJ Fiji. The mean total UCLA GIT score for the patients were calculated at week 0,2 and 16, as well as the mean staining per area of the duodenum biopsies at week 0,2 and 6 for all the markers. Finally, correlations between mean staining per area and mean total UCLA GIT score were assessed for ACHIM and placebo patient groups by Pearson correlation ( $r$ ).

Results: All nine patients included in the pilot trial were female and had limited cutaneous SSc. Groups receiving ACHIM and placebo had comparable disease duration (1). We found that relative change in staining per area for SIR from week 0 to week 2 differed between the ACHIM group and the placebo group (Figure $1 \mathrm{~A}$, left panel). Similar changes were observed for anti-gp38, anti-CD38 and anti-CD64 (left panel in Figures 1B-D). Strong correlations were found between mean total UCLA GIT score and the mean staining per area of the markers SIR ( $r=0.98)$, anti-gp38 ( $r=0.94)$, anti-CD34 ( $r=0.85)$ and CD64 ( $r=0.93)$ in the ACHIM group (Figure 1, A-D). In the placebo group, there was no correlation between the UCLA GIT score and anti-gp38 $(r=0.22)$ and anti-CD64 ( $r=0.21)$, however, a strong correlation were observed to $\mathrm{SIR}(\mathrm{r}=0.86)$ and anti-CD38 ( $r=0.92)$ staining (Figure 1A-D, right panel).

Conclusion: This explorative data set indicates different effects of FMT by ACHIM and placebo on the duodenal mucosa of SSc patients with GIT affection. Interestingly, we observed correlations between mucosal markers and improved patient reported GIT symptoms in the ACHIM group.

REFERENCES

[1] Fretheim H, Chung BK, Didriksen H, Bækkevold ES, Midtvedt Ø, Brunborg $\mathrm{C}$, et al. Fecal microbiota transplantation in systemic sclerosis: A double-blind, placebo-controlled randomized pilot trial. PLoS One. 2020;15(5):e0232739.
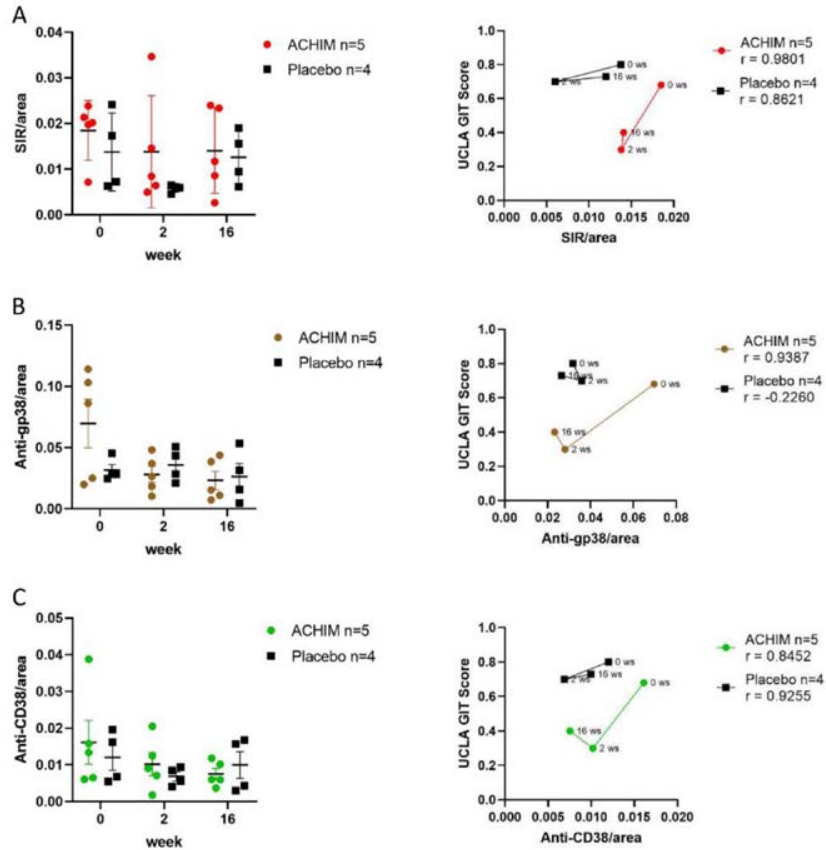

D
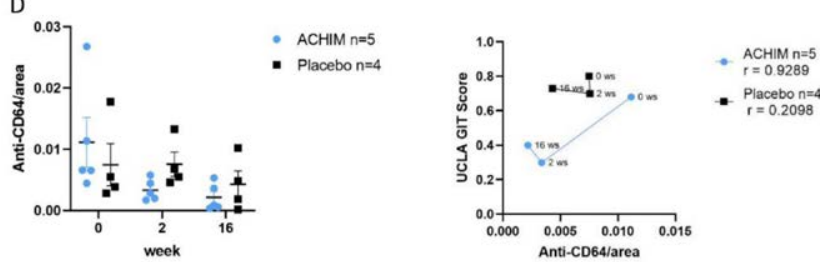

Figure 1. Relative staining per area in ACHIM and placebo group (left panel) and correlation between total UCLA GIT score and staining ratios at time point 0,2 and 16 weeks in patients receiving $\mathrm{ACHIM}$ and placebo (right panel).

Acknowledgements: We will like to thank Maria Comazzi for all the work she have done with the IHC stainings

Disclosure of Interests: Henriette Didriksen Speakers bureau: Travel bursary - GSK

Travel bursary and speaker - Actelion, Noemi Strahm: None declared, Øyvind Molberg: None declared, Håvard Fretheim Speakers bureau: Received travel bursaries from Actelion, and remuneration from Bayer., Torhild Garen: None declared, Øyvind Midtvedt: None declared, Oliver Distler Speakers bureau: Actelion, Kymera Therapeutics, Mitsubishi Tanabe Pharma, Abbvie, Acceleron, Alexion, Amgen, AnaMar Arxx Therapeutics, Baecon Discovery, Blade Therapeutics, Corbus Pharmaceuticals, Drug Development International Ltd, CSL Behring, Galapagos NV, Glenmark Pharmaceuticals, GSK, Horizon (Curzion) Pharmaceuticals, Inventiva, iQvia, Kymera Therapeutics, Lilly, Novartis, Pfizer, Topadur and UCB, Grant/research support from: Boehringer Ingelheim, Beyer, Gabriela Kania: None declared, Anna-Maria Hoffmann-Vold Speakers bureau: Actelion, Roche, Bayer, Merck Sharp \& Dohme, Lilly, ARXX and Medscape, Grant/research support from: Boehringer Ingelheim DOI: 10.1136/annrheumdis-2021-eular.2936

\section{\begin{tabular}{|l|l}
\hline POS0868 THE FORMULA TO PREDICT TACROLIMUS \\
\hline
\end{tabular} CONCENTRATION ACCORDING TO GENOTYPING OF CYP3A5 IS USEFUL FOR EFFECTIVE TREATMENT IN INTERSTITIAL LUNG DISEASE WITH DERMATOMYOSITIS}

M. Katayama ${ }^{1}$, S. Horibata ${ }^{2,3}$, S. Takahashi ${ }^{1,3}$, M. Takahashi ${ }^{3}$, T. Saito ${ }^{3}$, N. Amano ${ }^{1}$, K. Yoshida ${ }^{1}$, S. Hatachi ${ }^{1}$, K. Yorifuji ${ }^{2,3}$, S. Kumagai ${ }^{1,3}{ }^{1}$ Shinko Hospital, Center for Rheumatic Disease, Kobe, Japan; ${ }^{2}$ Shinko Hospital, Department of Pharmacy, Kobe, Japan; ${ }^{3}$ Shinko Institute for Medical Research, Kobe, Japan 